\title{
L'immunodiagnostic des lésions cancéreuses
}

Rosemonde Mandeville Professeur à l'université du Québec

De très nombreux anticorps monoclonaux, spécifiques de tissus, de lignées et de stades de différenciation, sont disponibles. D'ores et déjà utilisés pour le diagnostic et la surveillance de cancers, ils se révéleront peut-être d'un grand intérêt thérapeutique, "ciblant " des produits cytotoxiques vers les cellules tumorales à détruire.

\section{RÉFÉRENCES}

1. Köhler G, Milstein C. Continuous culture of fused cells secreting antibody of predetermined specificity. Nature $1975 ; 256$ : 495-7. 2. Stein H, Uchanska-Ziegler B, Gerdes J, Ziegler A, Wernet P. Hodgkin and SternbergReed cells contain antigens specific to late cells of granulopoiesis. Int $J$ Cancer $1982 ; 29$ : 283-90.

3. Habeshaw JA, Bailey D, Stansfeld AG Greaves MF. The cellular content of non Hodgkin lymphomas: A comprehensive analysis using monoclonal antibodies and other surface marker techniques. Br $J$ Cancer $1983 ; 47: 327-51$.

4. Lennert K, Stein H, Feller AC, Gerdes J. Morphology, cytochemistry and immunohistology of T-cell lymphomas. In : Vitetta ES, ed. $B$ and $T$ cell tumors. UCLA Symposia on Molecular and Cellular Biology, vol. XXIV. New York : Academic Press, 1982 ; 9-28. 5. Burns BF, Warnke RA, Doggett RS, Rouse RV. Expression of a T-cell antigen (Leu 1 ) by B-cell lymphomas. Am $J$ Pathol $1983 ; 113$ : 165-71.

6. Nathwani BN, Winberg CD, Diamond LW, Bearman RM, Kim H. Morphologic criteria for the differentiation of follicular lymphoma from florid reactive follicular hyperplasia : A -study of 80 cases. Cancer $1981 ; 48$ : 1794-806.

7. Wilson BS, Ruberto G, Ferrone S. Immunochemical characterization of a human high molecular weight-melanoma associated antigen identified with monoclonal antibodies. Cancer Immunol Immunother 1983 ; 14 : 196-201.

8. Steplewski Z, Koprowski H. Monoclonal antibody development in the study of colorectal carcinoma associated antigens. Methods In Cancer Research 1982 ; 22 : 286-316.

9. Schlom J, Wunderlich D, Teramoto YA. Generation of human monoclonal antibodies reactive with human mammary carcinoma cells. Proc Natl Acad Sci USA 1980; 77 6841-5.

$\mathrm{m} / \mathrm{s} n^{\circ} 1$ vol. 3 , janvier 87 es anticorps monoclonaux (AMC) sont des i m munoglobulines homogènes à la fois en terme d'espèce molécu-

plexes telles celles de la membrane cellulaire ou du cytosquelette de la cellule tumorale et permettent également l'étude de la tolérance particulière de l'organisme envers le tissu tumoral. Parallèlement, leur rôle dans le diagnostic de la maladie cancéreuse semble être de plus en plus évident et les perspectives de développement dans le domaine de la thérapie et de la prévention sont immenses. Nous voudrions présenter ici quelques applications de cette technologie en oncologie. essentiels par rapport aux immunsérums conventionnels : ils peuvent être produits en quantité quasi illimitée et avec des caractéristiques constantes, qu'ils soient dirigés contre un antigène courant ou rare. Depuis leur découverte [1], les AMC ont révolutionné des domaines aussi différents que l'immunologie, la microbiologie, la toxicologie et connaissent actuellement un plein essor en cancérologie. Ils sont devenus, en quelques années, un outil diagnostique indispensable permettant la détection, l'isolement et la quantification de toute une série de molécules biologiques mal connues jusqu'à maintenant. De plus, là où les techniques de dosage immunologique existaient déjà, les AMC ont permis de les perfectionner et d'étendre leurs possibilités.

Par ailleurs, les AMC ouvrent de nouveaux horizons dans l'analyse de structures antigéniques com-
Diagnostic des leucémies et lymphomes malins

Un nombre très important d'AMC identifiant des populations cellulaires différentes dans le sang humain a déjà été produit et caractérisé. Ces anticorps ont tout d'abord conduit à la caractérisation de " marqueurs " permettant d'identifier des populations cellulaires par la présence ou l'absence d'un antigène. Ensuite, des ateliers internationaux de nomenclature ont permis la définition des ensembles d'anticorps reconnaissant les mêmes cellules ou classes de différenciation $(\mathrm{CD}=$ clusters of differentiation) (Tableau I).

Utilisée d'abord pour la classification des anticorps antilymphocyte, cette méthode s'étend progressivement aux autres lignées hématopoiétiques et pourrait ultérieurement contribuer 


\section{RÉFÉRENCES}

10. Mandeville R, Lecomte J, Sombo FM, Chausseau JP, Giroux L. Production, purification and biochemical characterization of monoclonal antibodies reacting with human breast carcinoma cells. In : Chatterjee SN ed. Monoclonal Antibodies : Diagnostic and Therapeutic Use in Tumor and Transplantation. Littleton : PSG Publishing, 1985 : 63-71.

11. Ceriani RL, Sasaki M, Sussman $H$, Wara WM, Blank EW. Circulating human mammary epithelial antigens in breast cancer. Proc Natl Acad Sci USA 1982 ; 79 : 5420-4. 12. Mulshine JL, Cuttitta F, Bibro M, et al. Monoclonal antibodies that distinguish nonsmall cell from small cell lung cancer. $J$ Jmmunol 1983 ; 131 : 497-502.

13. Bast RC, Klug TL, St John E, et al. A radioimmunoassay using a monoclonal antibody to monitor the course of epithelial ovarian cancer. $N$ Engl J Med 1983 ; 309 : 883-7. 14. Cuttitta F, Rosen S, Gazdar AF, Minna JD. Monoclonal antibodies that demonstrate specificity for several types of human lung cancer. Proc Natl Acad Sci USA 1981; 78 : 4581-95.

15. Frankel AE, Rouse RV, Wang MC, Chu TM, Herzenberg LA. Monoclonal antibodies to a human prostate antigen. Cancer Res $1982 ; 42$ : 3714-8.

16. McGee Jo'D, Woods JC, Ashall F, Bramwell ME, Harris $H$. A new marker for human cancer cells. 2. Immunohistochemical detection of the $\mathrm{Ca}$ antigen in human tissues with the Ca1 antibody. Lancet 1982 ; ii : 7-11. 17. Chavanel G, Frenoy $\mathrm{N}$, Escribano $\mathrm{MJ}$, Burtin P. Production of monoclonal antibodies against the non-specific cross-reacting antigen (NCA). Oncodev Biol Med 1983 ; 4 : 209-17.

18. Krenner M. Diagnostic immunohistochimique des tumeurs ovariennes avec l'anticorps monoclonal OC 125. In : Chatal JF, Douis M, eds. Anticorps Monoclonaux en Cancérologie : Applications Cliniques. Nantes, Éditions Médicales Françaises, 1985 : 35-8.

19. Gatter KC, Abdulaziz Z, Beverly $P$ et al. Use of monoclonal antibodies for the histopathological diagnosis of human malignancy. $J$ Clin Pathol 1982 ; 35 ; 1253-67.

20. Coakham HB, Garson JA, Brownell B et al. Use of monoclonal antibody panel to identify malignant cells in cerebrospinal fuid. Lancet 1984 ; i : 1095-7.

21. Thirion $B$. Intérêt clinique du marqueur CA 19.9. Résultats d'une étude multicentrique. In : Chatal JF, Douis M, eds. Anticorps Monoclonaux en Cancérologie: Applications Cliniques. Nantes, Nouvelles Éditions Médicales Françaises, 1985 : 25-34

22. Epenetos AA, Canti G, TaylorPapadimitriou $J$, et al. Use of two epitheliumspecific monoclonal antibodies for diagnosis of malignancy in serous effusions. Lancet 1982 ; ii : 1004-6.

23. Dabelsteen E, Graem N, Hakomori SI, Young WW. Monoclonal antibodies in the diagnosis of epithelial premalignant lesions. Bull Cancer (Paris) 1983; $70: 127-31$.

24. Sears HF, Herlyn M, Del Villano B, Steplewski Z, Koprowski H. Monoclonal antibody detection of a circulating tumorassociated antigen : II. A longitudinal evaluation of patients with colorectal cancer. J Clin à la classification des cancers. En plus de la description des particularités phénotypiques de chaque variété de leucémies et lymphomes, les AMC sont d'un grand secours pour résoudre différents problèmes de diagnostic différentiel et déterminer le stade évolutif de la maladie, notamment entre leucémie et lymphome ou lymphome leucémique. Ces marqueurs permettent souvent de donner une idée du pronostic en raison de l'appartenance à un stade de maturité plus ou moins avancé (classification CD).

Détermination du phénotype des leucémies et des lymphomes malins. Il est actuellement possible de déterminer, à l'aide d'une gamme d'AMC, le phénotype de la plupart des lymphomes malins. Dans ces études comparatives, les différents AMC utilisés se servent mutuellement de témoin.

La majorité des lymphomes développés à partir des lymphocytes $\mathrm{B}$ expriment des immunoglobulines de surface (IgS) parfois associées à des Ig cytoplasmiques, notamment dans les immunocytomes et les formes immunoblastiques [2, 3]. Il s'agit le plus souvent d'IgM de type kappa ou lambda ou d'IgM + IgD. Cette dernière éventualité se rencontre couramment dans les leucémies lymphoïdes chroniques [2]. Les lymphomes à noyau clivé (centrocytique), possédant ces immunoglobulines, auraient un meilleur pronostic clinique. Dans certains lymphomes immunoblastiques ou lymphoblastiques, l'impossibilité de détecter des IgS peut rendre difficile le rattachement de la prolifération à la lignée B lymphocytaire. La mise en évidence d'un antigène commun aux cellules B (à l'exception des plasmocytes) par l'anticorps B1 ou B4 (CD19 et CD20) est alors d'un précieux secours [2] (Tableau I). Comme les follicules réactionnels, les follicules lymphomateux sont marqués par l'anticorps antiCALLA (CD10) [2, 3]

L'étude immunohistochimique des lymphomes $\mathrm{T}$ est plus difficile que celle des lymphomes $B$ en raison de l'absence de " marqueurs de monoclonalité " [4]. Les lympho- mes T cutanés ont le plus souvent un phénotype T-helper (CD4) et les lymphomes $\mathrm{T}$ de type lymphoblastique un phénotype complexe : $\mathrm{OKT}^{+}{ }^{+}, \mathrm{OKT}^{+}{ }^{+}$(CD4, CD8) ou OKT6 + (CD1). En revanche, les autres lymphomes $T$, notamment de type immunoblastique, ont des phénotypes plus variables : OKT4+(CD4), OKT8+ (CD8), ou $\mathrm{OKT}_{4}{ }^{+}$et $\mathrm{OKT} 8{ }^{+}(\mathrm{CD} 4$, CD8). Par ailleurs, T-helper néoplasique et $\mathrm{T}$-suppresseur réactionnel (ou vice versa) peuvent être intimement mêlés, et d'abondance parfois comparable, de telle sorte qu'il peut être délicat de déterminer le phénotype réel de la population tumorale.

Dans la maladie de Hodgkin, le granulome est souvent principalement constitué de lymphocytes Thelper (CD4) mais les cellules de Sternberg-Reed n'expriment que l'antigène HLA-DR. Récemment, Stein et coll. [2] ont fait état d'un nouvel anticorps (Ki-1) qui reconnaîtrait un antigène présent sur ces cellules. Ki-1 reconnaît aussi une population minoritaire des ganglions lymphatiques et de la moelle osseuse ; ces cellules sont considérées comme la contre-partie normale des cellules de SternbergReed.

Distinction entre lymphomes et pseudo-lymphomes. Devant une prolifération lymphoïde d'architecture diffuse, plusieurs diagnostics sont envisageables : pseudolymphome, lymphome lymphocytique (correspondant le plus souvent à une leucémie lymphoïde chronique), lymphome lymphoplasmocytaire, lymphome centrocytique ou lymphome $\mathrm{T}$. La mise en évidence d'IgS monoclonales, parfois associées à des Ig cytoplasmiques, assure le diagnostic de lymphome B. Ici, l'antigène $T 65$ mérite une mention particulière. Il s'agit d'un antigène identifiable par plusieurs AMC (T 101, Leu 1, OKT1), normalement présent sur la majorité des lymphocytes $T$ normaux ou néoplasiques et peut-être sur une population très minoritaire de lymphocytes B [5]. Cependant, les cellules des leucémies lymphoïdes chroniques B et des lymphomes centrocytiques (diffus ou fol- 
liculaires et diffus) expriment fréquemment cet antigène. Ainsi, une prolifération formée de petites cellules lymphoïdes Leu $1^{+}$ (ou $\mathrm{T}_{101^{+}}$) mais Leu $4^{-}$ou (OKT3-), donc $\mathrm{CD}^{-}{ }^{+}$et $\mathrm{CD}^{-}{ }^{-}$, aura très probablement un caractère néoplasique [5]. Ceci peut être utile lorsqu'un bruit de fond trop important gêne la mise en évidence de divers anticorps dirigés contre les IgS.

Devant une prolifération lymphoïde d'architecture folliculaire, on peut diagnostiquer une hyperplasie réactionnelle ou un lymphome folliculaire, notamment de forme dite intrafolliculaire. Malgré les critères récemment proposés par Nathwani et coll. [6], il reste des cas difficiles qui ne peuvent être résolus qu'à l'aide de l'immunohistochimie sur coupe à congélation. Le premier critère attestant de la nature lymphomateuse des follicules est la présence d'IgS monotypiques. Le second critère est l'absence, à l'intérieur des follicules lymphomateux, d'Ig polyclonales extra-cellulaires, habituellement présentes dans les follicules réactionnels.

\section{Diagnostic \\ des tumeurs solides}

Diagnostic immunopathologique. Les applications des anticorps monoclonaux en immunopathologie sont aujourd'hui si nombreuses qu'il est impossible d'en dresser un inventaire complet. Nous choisirons donc quelques exemples illustrant l'évolution rapide de cette technologie. Plusieurs AMC ont été préparés [7-15] par immunisation de souris avec des cellules entières, parfois aussi avec des préparations membranaires obtenues à partir de biopsies de tumeurs prélevées chirurgicalement. Parmi les antigènes nouveaux que les $\mathrm{AMC}$ ont permis de découvrir, certains sont des antigènes d'organes exprimés aussi bien dans la tumeur que dans le tissu normal [7]. D'autres semblent être réellement des antigènes associés aux tumeurs, encore que ce caractère doive toujours être affirmé avec prudence.

Tableau I

PRINCIPAUX AMC RECONNAISSANT DES ANTIGÈNES

DE SURFACE DE CELLULES HÉMATOPOÏÉTIQUES. CLASSIFICATION INTERNATIONALE

\begin{tabular}{|c|c|c|}
\hline $\begin{array}{l}\text { Numéro } \\
\text { du cluster }\end{array}$ & Cellules reconnues & $\begin{array}{l}\text { Anticorps } \\
\text { les plus couramment utilisés }\end{array}$ \\
\hline CD1 & Corticothymocytes & OKT6, IOT6, T6, Leu 6 \\
\hline CD2 & $\begin{array}{l}\text { Lymphocytes } T \text { formant des } \\
\text { rosettes mouton }\end{array}$ & OKT11, IOT11, T11, Leu 5b \\
\hline $\mathrm{CD3}$ & Lymphocytes T, Pan-T & OKT3, IOT3, Leu 4 \\
\hline $\mathrm{CD} 4$ & T-helper/inducer & OKT4, IOT4, Leu 3a \\
\hline CD5 & $\begin{array}{l}\text { Lymphocytes } \mathrm{T}+\text { sous-popula- } \\
\text { tions B }\end{array}$ & OKT1, IOT1, T1A, Leu 1 \\
\hline CD8 & T-cytotoxique/suppresseur & OKT8, IOT8, T8, Leu 2a \\
\hline CD10 & $\begin{array}{l}\text { Pré-B, polynucléaire, Ag commun } \\
\text { des leucémies aiguês lymphoblas- } \\
\text { tiques }\end{array}$ & CALLA, T5, IOT5a \\
\hline CD11 & Monocytes et Granulocytes & Mo1, IOM1, Leu M5 \\
\hline CD16 & Récepteurs pour les fragments Fc & Leu 11 \\
\hline $\mathrm{CD} 18$ & Lymphocytes T, B et monocytes & IOT16 \\
\hline CD19 & Lymphocytes B & B4 \\
\hline $\mathrm{CD} 20$ & Lymphocytes B & IOB1, B1 \\
\hline CD24 & LAL, cellules B, granulocytes & 1OB3 \\
\hline $\mathrm{CD} 25$ & $\begin{array}{l}\text { T activés in vitro (mitogènes) ou } \\
\text { in vivo }\end{array}$ & TAC, anti-IL-2, IOM4, OKT26a \\
\hline
\end{tabular}

Série Leu (Becton Dickinson Co. Mountain View, CA), Série T (Ortho Pharmaceutical Corporation. Rariran, NJ), Pan B (Dako, Lab.), R4/23 (D.Y. Mason, Oxford). Série Lyt (New England Nuclear Co., Boston, Ma)

En effet, on retrouve parfois ces antigènes dans quelques types de cellules normales et un certain nombre de ceux-ci possède un caractère onco-fœtal [8]. De plus, il existe un nombre plus restreint d'AMC qui semblent reconnaître des antigènes (comme les antigènes CA et NCA) communs à une grande variété de cancers $[16,17]$. Citons ici l'apport de deux AMC, OC 125 [13] et 19.9 [8], dans la précision du profil antigénique des tumeurs ovariennes d'origine épithéliale. L'anticorps monoclonal OC 125 réagit avec un déterminant antigénique, le CA 125, qui est associé à la plupart des tumeurs ovariennes non muqueuses. Ainsi, $82 \%$ des tumeurs épithéliales séreuses sont positives tandis que toutes les tumeurs muqueuses sont négatives. Lorsque les tumeurs séro-muqueuses sont positives, c'est toujours la composante séreuse qui est marquée tandis que la composante muqueuse est négative. Les tumeurs de l'endomètre expriment le CA 125 dans $63 \%$ des cas et seulement $25 \%$ des tumeurs à cellules claires sont positives. Les tumeurs de Brenner et tous les carcinomes indifférenciés sont négatifs [14]. D'autre part, dans les tumeurs muqueuses où le $\mathrm{CA} 125$ est régulièrement négatif, le $\mathrm{CA}$ 19.9 se présente comme un bon marqueur puisque $84 \%$ des tumeurs étudiées sont positives avec cet anticorps. Le CA 125 se présente donc comme un bon marqueur des tumeurs épithéliales séreuses de l'ovaire, alors que le CA 19.9 est un bon marqueur des tumeurs épithéliales muqueuses.

Ces deux antigènes recouvrent presque la totalité de la pathologie ovarienne épithéliale [14].

A cause de ce polymorphisme, l'utilisation d'une "batterie " bien choisie d'AMC (dont les épitopes sont parfaitement connus) semble être plus utile que l'utilisation 


\section{RÉFÉRENCES}

25. Goldenberg DM, DeLand HF. History and status of tumor imaging with radiolabeled antibodies. Journal Biological Response Modifiers $1982 ; 1: 121-36$

26. Epenetos AA, Britton KE, Mather S, et al. Targeting of iodine-123-labelled tumorassociated monoclonal antibodies to ovarian, breast and gastrointestinal tumors. Lancet 1982 ; ii : 999-1003.

27. Larson SM, Brown JP, Wright PW, et al. Imaging of melanoma with I-131 labeled monoclonal antibodies. $J$ Nucl Med 1983 ; 24 : 123-9. 28. Moldof sky PJ, Sears HF, Mulhern CB, et al. Detection of metastatic tumor in normalsized retroperitoneal lymph nodes by monoclonal antibody imaging. $N$ Engl $J$ Med 1984 ; 311: 106-7.

29. Scheinberg DA, Strand M, Ganson OA. Tumor imaging with radioactive metal chelates conjugated to monoclonal antibodies. Science 1982 ; $215: 1511-3$

30. Rainsbury RM, Ott RJ, Westwood JH, et al. Location of metastatic breast carcinoma by a monoclonal antibody chelate labelled with Indium-111. Lancet 1983 ; ii : 934-8.

31. Vaughan ATM, Bateman WJ, Fisher DR. The in vivo fate of a ${ }^{211}$ A t labelled monoclonal antibody with known specificity in a murine system. Int J Radiat Oncol Biol Phys $1982 ; 8$ : 1943-6.

32. Wahl RL, Parker CW, Philpott GW. Improved radioimaging and tumor localization with monoclonal $\mathrm{F}$ (ab') $2 . J$ Nucl Med 1983 ; 24 : 316-25.

33. Moldofsky PJ, Powe J, Mulhern CB, et al. Metastatic colon carcinoma detected with radiolabelled $\mathrm{F}\left(\mathrm{ab}^{\prime}\right)_{2}$ monoclonal antibody fragments. Radiology 1983 ; 149 : 549-55.

34. Miller RA, Maloney D, Warnke R, et al. Considerations for treatment with hybridoma antibodies. Progress in Cancer Research and Treatment $1982 ; 21$ : 133-46.

35. Ritz J, Pesando JM, Sallan SE et al. Serotherapy of acute lymphoblastic leukemia with monoclonal antibody. Blood $1981 ; 58$ : 141-52. 36. Gillilland DG, Steplewski Z, Collier RJ, Mitchell KF, Chang TH, Koprowski H. Antibody directed cytotoxic agents : use of monoclonal antibodies to direct the action of toxin A chains to colorectal carcinoma cells. Proc Natl Acad Sci USA 1980 ; 77 : 4539-43.

37. Tsukada Y, Hurwitz E, Kashi R at al. Chemotherapy by intravenous administration of conjugates of daunomycin with monoclonal and conventional anti-rat $\alpha$-f ctoprotein antibodies. Proc Natl Acad Sci USA 1982 ; 79 : 7896-9. 38. Bernhard MI, Foon KA, Oeltmann TN, et al.. Guinea pig line 10 hepatocarcinoma model : characterization of monoclonal antibody and in vivo effect of unconjugated antibody and antibody conjugated to diphteria toxin A chain. Cancer Res 1983 ; 43 : 4420-8.

39. Filpovich $A H$, Youle RJ, Neville DM Jr, Vallera DA, Quinones RR, Kersey JH. Exvivo treatment of donor bone marrow with anti T-cell immunotoxins for prevention of graftversus host disease. Lancet 1984; i : 469-72. 40. Ritz J, Salan JE, Bast RC, Lipton JM, Nathan DG, Schlossman SF. Autologous bone marrow transplantation in Calla-positive acute lymphoblastic leukemia after in vitro treatment with J5 monoclonal antibody and complement. d'un seul AMC. De plus, la superposition des réactivités ou la comparaison des réactivités de plusieurs anticorps donne souvent des résultats plus précis. Plusieurs travaux ont donc permis : (a) d'établir avec certitude l'origine néoplasique de certaines tumeurs humaines [19] ; (b) de différencier les tumeurs d'origine ovarienne de celles d'origine gastrointestinale [13] ; (c) de préciser la localisation de la tumeur primaire inconnue lors du diagnostic initial d'un foyer métastatique adénocarcinomateux ou dans des cas de méningites carcinomateuses [20, 21] ; et enfin (d) de dépister la présence des cellules cancéreuses dans les effusions pleurales [22] et les biopsies de moelle [23].

Dépistage précoce des lésions cancéreuses. Les AMC permettent également un dépistage précoce dans les populations à haut risque de développer un cancer. Ainsi, la détection précoce de lésions précancéreuses pour un nombre croissant de tumeurs (poumons, lèvres, utérus, sein) ouvre des perspectives prometteuses débouchant sur une véritable prévention.

Études cytologiques : la cytologie permet de déceler les premières anomalies cellulaires témoignant d'une transformation maligne. Sur des sujets à symptomatologie d'appel, la cytologie constitue la première étape d'un diagnostic précoce pour certains cancers, tels que ceux des bronches, de la cavité buccale, de la vessie et de la prostate. Ainsi, Dabelsteen et coll. [23] rapportaient récemment l'étude immunocytochimique des lésions dysplasiques buccales en utilisant des AMC qui réagissent avec les précurseurs des antigènes des groupes sanguins $A$ et $B$, soit l'antigène $H$ à chaîne de type 2 ou son précurseur, la $\mathrm{N}$ acétyllactosamine. Ces deux antigènes sont localisés sur la membrane des cellules de l'épithélium basal et sur les cellules à épines et granuleuses de la peau. L'accumulation de l'antigène $\mathrm{H}$ à chaîne de type 2 dans les lésions précancéreuses de la bouche et la perte du même antigène dans les lésions précancéreuses de la peau a per- mis le diagnostic précoce des cancers épithéliaux.

Dosage biologique des marqueurs tumoraux : le dosage régulier de certains antigènes dans le sérum a permis un progrès notable, soit au stade de confirmation du diagnostic, soit dans la surveillance postthérapeutique [21, 24]. Quand on observe une élévation significative et persistante d'un de ces marqueurs, on peut le plus souvent en déduire la survenue d'une récidive. Cela est vrai, par exemple, pour la détection de l'antigène CA 19.9 dans les cas de cancers du pancréas exocrine (sensibilité $89 \%$, spécificité $97 \%$ ). Dans les cas de cancers colo-rectaux, le dosage simultané de l'antigène CA 19.9 et de l'antigène carcinoembryonnaire permet de déceler environ $50 \%$ des tumeurs des stades $\mathrm{C}$ de la classification de Dukes, plus de $86 \%$ des récidives ou des stades $D$ sans atteinte hépatique et enfin plus de $90 \%$ des tumeurs ou des récidives avec atteinte hépatique. Dans les cancers des voies biliaires, le dosage du CA 19.9 semble particulièrement sensible (11 cas, tous positifs) [21].

Le taux sérique de l'antigène CA 125 s'élève dans $80 \%$ des cas de tumeurs séreuses de l'ovaire $[13,18]$. Dans $93 \%$ des cas, le changement détecté au cours du suivi semble être en relation directe avec le pronostic clinique. De plus, des études préliminaires démontrent que cet antigène permet de prédire la présence de récidives dans $80 \%$ des cas, et ceci six à sept mois avant leur détection clinique.

Le radiomarquage. Les $\mathrm{AMC}$, couplés à une substance de contraste ou à un radioisotope, sont susceptibles de raffiner le pouvoir diagnostique des techniques non invasives comme la tomographie axiale numérique ou la scintigraphie isotopique. Cette technique permettrait d'évaluer plus spécifiquement l'étendue de la masse tumorale ou la présence de micrométastases [25-28]. Cependant, les premiers résultats ont été très controversés et des techniques de soustraction sont souvent nécessai- 
res pour minimiser le bruit de fond. Afin d'améliorer le rendement de cette technique, on utilise à l'heure actuelle des radioéléments qui possèdent une courte demi-vie et dont le rayonnement gamma est de faible énergie, et/ou on conjugue les AMC à des chélates bifonctionnels avant de les marquer avec des isotopes. Parmi ceux-ci, citons : le scandium-47, le gallium-67, le technetium-99m, l'indium-111 [29, 30] et l'astatine-211 [31]. En effet, ces complexes permettent de minimiser les échanges avec les protéines plasmatiques. De plus, ces complexes sont très rapidement excrétés par voie rénale s'il y a rupture de la liaison chélate/anticorps. Récemment, plusieurs auteurs rapportent que l'utilisation de l'anticorps sous forme de fragment $F\left(a b^{\prime}\right)_{2}$ est de loin plus efficace que l'utilisation de la molécule entière d'immunoglobuline [32, 33].

\section{Stratégies opérationnelles}

L'utilisation des AMC représente à l'heure actuelle une technique d'avenir pour le diagnostic et le traitement des cancers. L'état actuel de la recherche et des applications cliniques des AMC permet la définition de plusieurs stratégies opérationnelles. Ainsi, des AMC reconnaissant :

- des antigènes de tumeurs (exantigènes onco-fœtaux), pourraient servir au diagnostic des lésions cancéreuses,

- des antigènes de différenciation, des précurseurs immatures, seraient utiles pour le pronostic comme pour le suivi et le diagnostic précoce de rechutes des maladies cancéreuses,

- des antigènes de lignée (surface et cytosquelette), seraient utiles pour un diagnostic différentiel d'un tissu métastatique atypique.

Les AMC sont aussi potentiellement utilisables en thérapeutique du cancer. Il est bien démontré actuellement qu'en l'absence d'antigènes circulants, les AMC se lient rapidement et spécifiquement aux cellules tumorales. Même si le pouvoir cytotoxique de ces anti$m / s n^{\circ} 1$ ool. 3, janvier 87 corps (immunothérapie passive) ne suffit pas à éliminer les cellules tumorales, ceux-ci apparaissent comme un véhicule idéal pour amener un agent toxique au sein de la tumeur. Différentes substances antinéoplasiques peuvent être couplées aux AMC : les agents chimiothérapeutiques (immunodrogues), les liposomes porteurs de drogues ou certaines toxines comme la chaîne $\mathrm{A}$ de la ricine (immunotoxines). Les AMC pourront ainsi améliorer les performances d'une drogue : meilleure efficacité sur les cellules malignes, tout en évitant les effets toxiques dans les tissus sains. Il reste cependant à vaincre d'importantes difficultés, notamment : l'accessibilité des cellules malignes dans une masse tumorale ; l'induction par sélection de variants génétiques résistant à la drogue liée (toxines ou agents antinéoplasiques) ou dépourvus d'antigène-cible ; la présence dans le milieu intérieur (circulation) d'antigènes libérés par les cellules lysées. De plus, la modulation antigénique et la production d'anticorps anti-idiotypes demeurent à l'heure actuelle des problèmes difficiles à résoudre.

Mentionnons en dernier lieu que certains AMC sont à l'heure actuelle utilisés dans les " purges de moelle ", traitement in vitro de la moelle dans le cas de greffes allogéniques et autologues. Les objectifs de la purge médullaire diffèrent selon le type de greffes. Dans les cas de greffes de moelle autologues (où l'on utilise la propre moelle du sujet prélevée et cryopréservée avant radiochimiothérapie), ce traitement vise à l'élimination des cellules tumorales contenues dans le greffon. Dans les greffes allogéniques, le traitement est destiné à éliminer du greffon tout (ou partie) des lymphocytes $\mathrm{T}$ normaux présumés responsables de la GVH (Graft Versus Host Disease). Différentes méthodes ont été mises au point pour détruire les cellules cibles identifiées par l'AMC, notamment la cytolyse dépendant du complément, les immunotoxines ou les méthodes immunophysiques (couplage des AMC à des supports inertes ou métalliques)

\section{Summary}

Monoclonal antibodies elicited to surface antigens on a wide variety of normal and neoplastic cell types have been used with increasing frequency in the immunodiagnosis and classification of cancer. Their widest application so far has been in the in vitro analysis of leukemia and lymphoma using blood or bone marrow samples or tissue biopsy specimens. More recently, these antibodies were produced against solid tumorassociated antigens. The antibodies have helped to identify the tissue of origin of malignant neoplasms and, in some cases, to monitor the course of disease and/or to diagnose premalignant lesions. Carefully chosen panels of monoclonal antibodies are becoming very rapidly a valuable addition to the pathologist's diagnostic arsenal.

\section{ADRESSE ET TIRÉS À PART}

R. Mandeville : centre de recherche en immunologie, institut Armand-Frappier, 531, boulevard des Prairies, Laval-des-Rapides (Québec), Canada, H7V 4 Z3. 